\title{
Una estrategia de ecodiseño de piezas obtenidas mediante moldeo a presión: análisis microestructural aplicado a la desmaterialización ${ }^{(\bullet)}$
}

\author{
B. Suárez-Peña* y J. Asensio-Lozano**
}

\begin{abstract}
Resumen
En este trabajo se estudia la posibilidad de empleo de nuevas aleaciones de aluminio con estructuras micrográficas optimizadas que garanticen mejoras en el comportamiento mecánico de componentes fabricados mediante fundición a presión. El objetivo ha sido explorar la posibilidad de fabricación de ecopeldaños de elevación con destino a escaleras mecánicas, menos reforzados estructuralmente y por tanto más ligeros que los que hasta ahora se venían fabricando. Los resultados experimentales muestran que es factible disminuir el uso de materiales durante la fabricación y reducir, por tanto, el impacto a lo largo de la vida de los componentes, todo ello manteniendo o incluso mejorando las prestaciones en servicio y la seguridad.
\end{abstract}

\section{An ecodesign strategy for high pressure die casting components: microstructural analysis applied to mass reducing processes}

\begin{abstract}
In this work the study focused on the possibility of use of new aluminium alloys with optimized microstructures that ensure the mechanical properties requested for cast components made by high pressure die casting. The objective was to check the possibility of manufacture of structurally sound eco-steps for escalators with reduced structural integrity. The former arises as a result of a new redesign of the traditional steps aiming at a significant weight reduction. The experimental results show that it is feasible to cut the use of materials during processing and therefore to reduce the impact of the components during its lifetime, whilst the performance and safety standards are kept identical or even improved.
\end{abstract}

Keywords

Mass reducing processes; Mechanical properties; Attributive analysis; High-pressure die casting.

\section{INTRODUCCIÓN}

Actualmente, la evolución de la tecnología pone al alcance de las empresas nuevos medios con los que conseguir una importante reducción del consumo de recursos y mejorar la eficiencia de los equipos e instalaciones, todo ello, manteniendo las prestaciones y los procesos productivos y disminuyendo los costes económicos. El ecodiseño engloba al conjunto de acciones orientadas a la mejora medioambiental de un producto, en la etapa inicial de diseño. Incluye estudios de evaluación de energía y de materia utilizadas, así como de los residuos generados. Estos análisis permiten establecer las pautas a la hora de realizar la selección de los materiales utilizados en la elaboración de un producto, consiguiendo reducir el peso de los componentes y en su caso el número de éstos ${ }^{[1]}$.

La técnica de fundición a presión es una de las más utilizadas en la fabricación de componentes de aluminio ${ }^{[2]}$. La industria del moldeo a presión del aluminio provoca numerosos problemas ambientales, tales como emisiones, aguas residuales, etc. ${ }^{[3]}$. Recientemente, Neto et al. ${ }^{[4-5]}$ han desarrollado modelos y estrategias de reducción de impacto medioambiental aplicados a plantas de procesado de aluminio mediante fundición a presión. Por otro lado, Choi et al. ${ }^{[6]}$, en sus trabajos de investigación, concluyen que el diseño del producto, la selección del material y el método de fabricación,

\footnotetext{
(•) Trabajo recibido el día 27 de junio de 2008 y aceptado en su forma final el día 19 de enero de 2008.

* Departamento de Ciencia de los Materiales e Ingeniería Metalúrgica, E.U.I.T.I., Carretera de Castiello de Bernueces, s/n, Campus de Viesques, Universidad de Oviedo, 33203 Gijón (Asturias). ESPAÑA.

${ }^{* *}$ Departamento de Ciencia de los Materiales e Ingeniería Metalúrgica, E.T.S. Ingenieros de Minas, C/ Independencia, 13, Universidad de Oviedo, 33004 Oviedo (Asturias). ESPAÑA.
} 
son los factores críticos causantes del impacto medioambiental. A la vista de lo anterior, el presente trabajo surge como un proyecto de desmaterialización en el que la estrategia de ecodiseño se centra en aligerar el peso de peldaños de elevación de escaleras mecánicas. Se trata de piezas con paredes finas, pero que requieren buena resistencia, buena precisión dimensional y estrechas tolerancias, utilizándose en su fabricación la técnica de moldeo a presión. El reto tecnológico consiste en conseguir un componente que tras una reducción en su peso de un $20 \%$, fruto de un rediseño geométrico, mantenga idénticas ó mejores prestaciones que el que tradicionalmente viene fabricándose, y sin embargo pueda obtenerse sin modificación del proceso productivo.

El objetivo de este trabajo ha sido explorar la capacidad de fabricación de ecopeldaños de elevación con destino a escaleras mecánicas. Para ello, se han empleado dos aleaciones del sistema $\mathrm{Al}-\mathrm{Si}$, con presencia de microaleantes. Tras verificar, mediante ensayos metalográficos y mecánicos, la calidad del componente y de la aleación, un análisis atributivo permitió constatar el óptimo comportamiento de los peldaños.

\section{PROCEDIMIENTO EXPERIMENTAL}

En la producción de los peldaños de elevación convencionales (Fig. 1) se viene utilizando la aleación comercial Al-Si 12 (413.0) [7], cuya composición se detalla en la tabla I (aleación 1). Trabajos de investigación previos ${ }^{[8]}$ señalan como las más adecuadas para la realización de las pruebas de desmaterialización de peldaños, aquellas aleaciones afinadas con titanio y modificadas con estroncio. Bajo estas directrices, en el presente estudio se optó por la aleación 1 para fabricar los peldaños convencionales, mientras que para los ecopeldaños se eligió la aleación comercial preafinada y con modificación permanente denominada aleación 2. Los peldaños se fabricaron siguiendo el procedimiento industrial consignado en trabajos anteriores ${ }^{[9]}$, consistente en un desgasificado con nitrógeno previo a la inyección en la cámara y que se esquematiza en la figura 2.

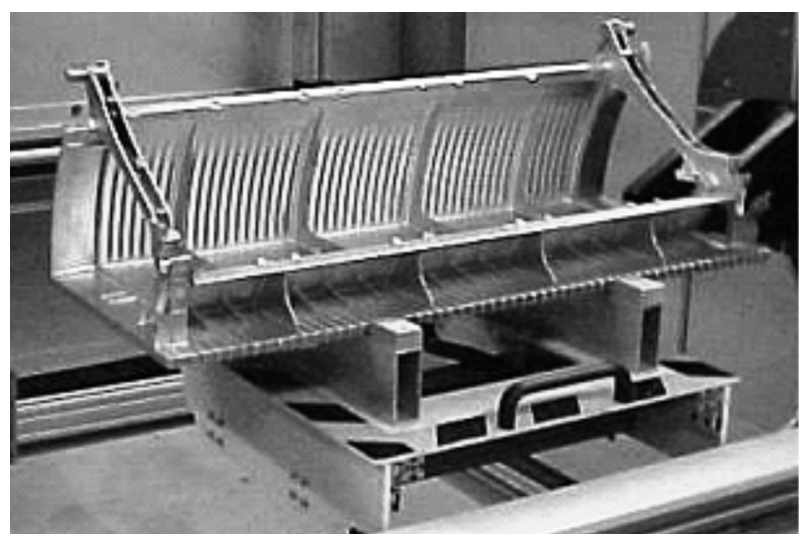

Figura 1. Peldaño de elevación.

Figure 1. Escalator step.

\subsection{Caracterización metalográfica de los peldaños de elevación}

Para llevar a cabo la caracterización metalográfica, se obtuvieron muestras de ambas aleaciones después de realizar la inyección. La preparación de las probetas metalográficas consistió en un desbaste con agua, empleando discos de carburo de silicio; un pulido con paños y pasta de diamante de 9,3 y 1 micras seguido de una etapa final de pulido con silicagel. Por último, se procedió al ataque, empleándose como reactivo una solución acuosa de $\mathrm{HF}$ al 0,5 \%, adecuada para la observación de fases por microscopía óptica (M. O.). El equipo utilizado ha sido un banco metalográfico NIKON EPIPHOT.

El empleo de las técnicas de la metalografía cuantitativa como el contaje de puntos, el análisis areal y la longitud media interceptada ${ }^{[10-13]}$, permitió obtener las medidas de la fracción en volumen y del tamaño de la fase de Al- $\alpha$ primaria $\left(\overline{\mathrm{A}}_{\alpha}\right)$ y de los cuboides de silicio $\left(\overline{d_{p}}\right)$. Las fracciones en volumen $\left(\mathrm{V}_{\mathrm{V}}\right)$ de ambas fases se calcularon tras contar $200 \mathrm{ca}$ racterísticas ó, en su defecto, evaluar éstas en 25 micrografías, mientras que el tamaño de las partículas se calculó tras medir 500 características ó, en su defecto, analizar 25 micrografías.

Tabla I. Composición química de las aleaciones comerciales en \% en peso

Table I. Chemical composition of commercial alloys expressed in weight-per cent

\begin{tabular}{cccccccccccc}
\hline Elemento (\%) & $\mathrm{Si}$ & $\mathrm{Fe}$ & $\mathrm{Cu}$ & $\mathrm{Mn}$ & $\mathrm{Mg}$ & $\mathrm{Ni}$ & $\mathrm{Zn}$ & $\mathrm{Pb}$ & $\mathrm{Ti}$ & $\mathrm{Sr}$ & $\mathrm{Cr}$ \\
\hline Aleación 1 & 12,90 & 0,82 & 0,04 & 0,20 & 0,01 & 0,006 & 0,03 & 0,02 & 0,008 & 0,00 & 0,013 \\
Aleación 2 & 9,50 & 0,63 & 0,05 & 0,32 & 0,29 & 0,007 & 0,03 & 0,008 & 0,12 & 0,03 & 0,003 \\
\hline
\end{tabular}




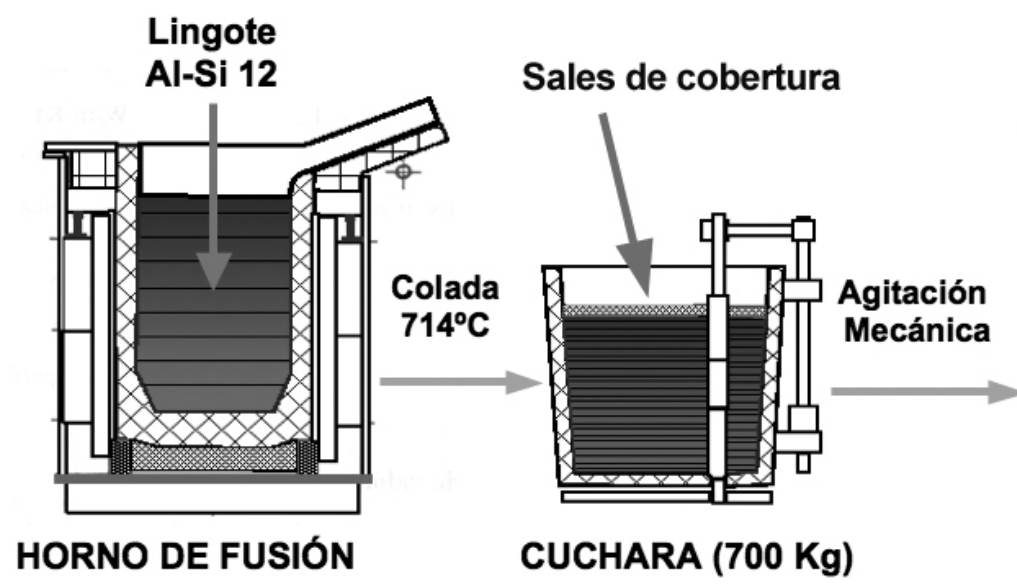

\section{TRATAMIENTO EN CUCHARA}
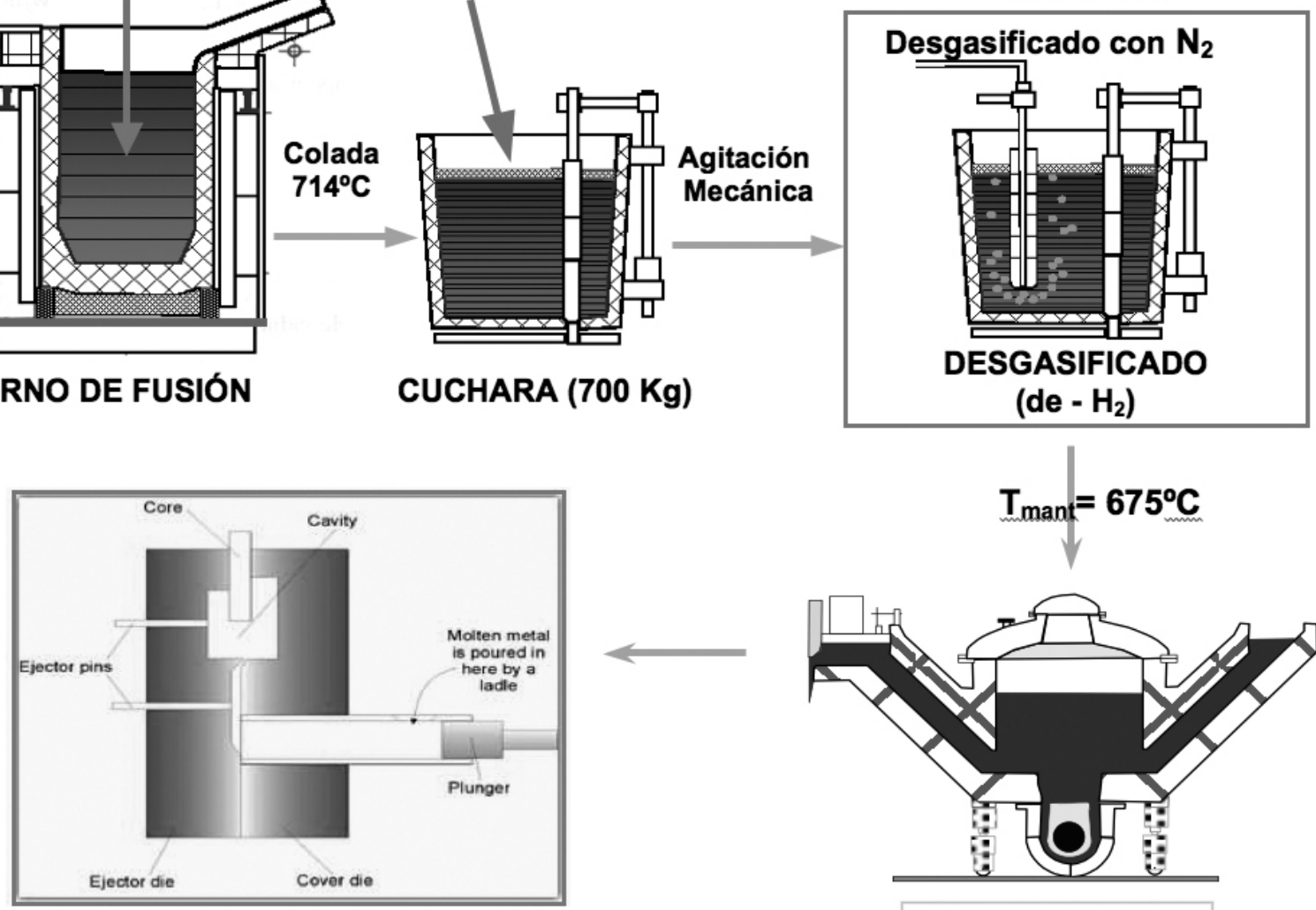

COLADA A PRESIÓN
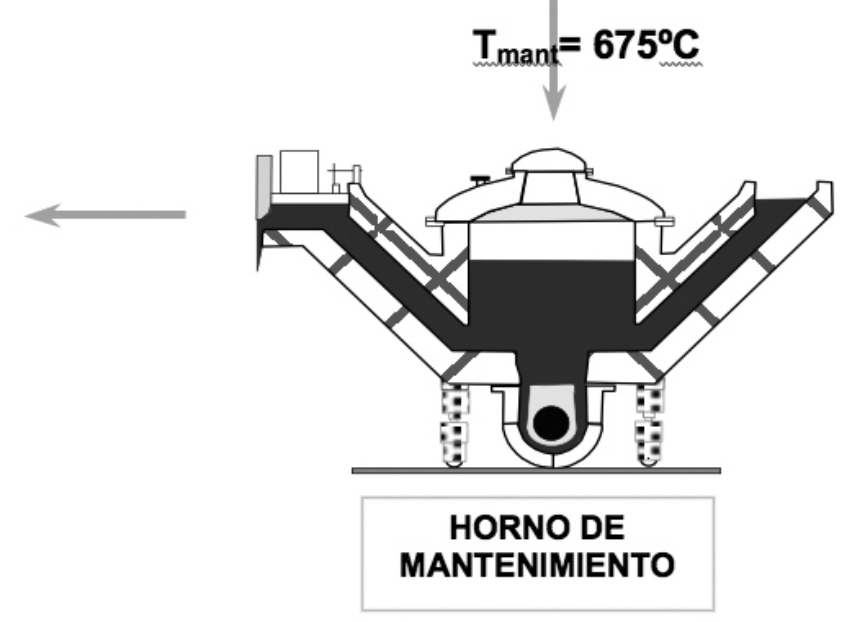

Figura 2. Procedimiento industrial de fabricación de los peldaños mediante colada a presión.

Figure 2. Industrial process for manufacture of escalator steps by high pressure die casting.

\subsection{Ensayo de tracción}

Tras la inyección, se apartaron dos peldaños de elevación convencionales (aleación 1) y dos ecopeldaños (aleación 2). Seccionando su nervio central, se extrajeron probetas de tracción de sección rectangular (100 $\mathrm{mm}$ de longitud, $16 \mathrm{~mm}$ de ancho y $4 \mathrm{~mm}$ de espesor). Estas probetas se obtuvieron por mecanizado longitudinal con el objeto de alcanzar un acabado superficial óptimo y paralelismo entre caras. Posteriormente, se ensayaron a tracción a temperatura ambiente, siguiendo la norma ASTM E8 ${ }^{[14]}$. Se empleó una maquina de ensayos INSTRON modelo 5582 , con una célula de carga de $10 \mathrm{kN}$, una velocidad de desplazamiento del cabezal móvil de $6 \mathrm{~mm} / \mathrm{min}$ y un extensómetro de $50 \mathrm{~mm}$ de longitud calibrada. Las propiedades mecánicas determinadas en todos los casos fueron: límite elástico $\left(R_{P}\right)$, resistencia a la tracción $\left(R_{m}\right)$ y alargamiento a la rotura $\left(A_{T}\right)$. Se realizaron dos ensayos por cada tipo de peldaño, y se tomó la media de ambos como resultado final.

\subsection{Ensayo de flexión en tres puntos}

Los ensayos de flexión se llevaron a cabo en peldaños individuales completos. Para ello se empleó un banco de ensayo normalizado que por aplicación consecutiva de esfuerzos-deformaciones, pretende derivar su comportamiento en fatiga de bajo número de ciclos, con superación del límite elástico (con deformación permanente) en cada ciclo (Fig. 3).

El ensayo consta de dos fases:

1. Fatiga por flexión de bajo número de ciclos Tras colocar el peldaño sobre los soportes de la máquina (Fig. 3), se carga éste en el centro con una fuerza estática de $1 \mathrm{kN}$, aplicándola verticalmente respecto a la superficie de pisada. Tras retirar la carga y medir la deformación provocada, se repite el ciclo con cargas de 3, 6, 10 y $15 \mathrm{kN}$. 2. Ensayo de rotura por flexión

Sin retirar el peldaño de su ubicación previa, tras el referido proceso de fatiga de la etapa anterior, se conduce el ensayo estático de flexión, aplicando 


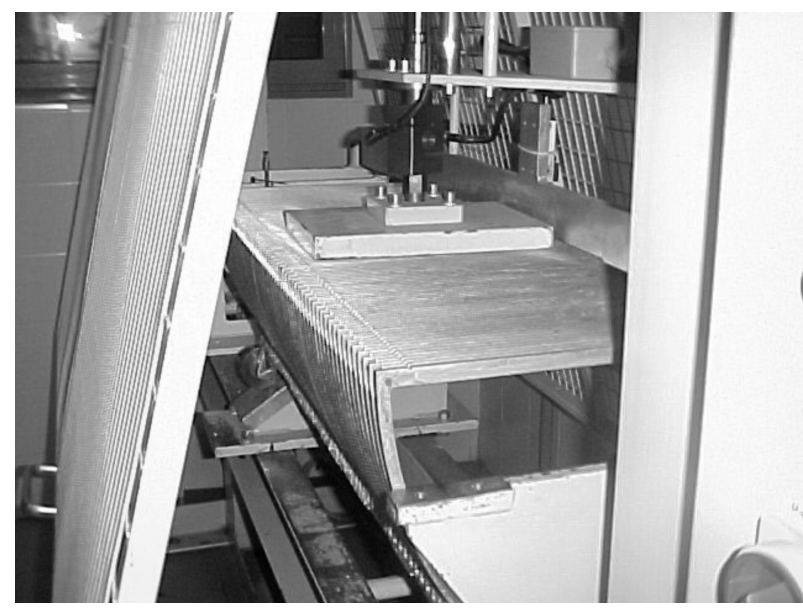

Figura 3. Banco para ensayo de flexión de peldaños de elevación.

Figure 3. Bend test bench for full-size testing of escalator steps.

una carga centrada hasta provocar la rotura del componente. Se anota la fuerza máxima $\left(F_{R}\right)$ y la flecha a rotura $\left(\delta_{R}\right)$.

El equipo de ensayo proporciona una curva fuerza-deformación (Fig. 4), que permite calcular la energía consumida en la rotura del material $\left(\mathrm{U}_{\mathrm{T}, \mathrm{b}}\right)$ a partir del área subtendida bajo la curva $\mathrm{F}-\delta$, como medida de su tenacidad máxima.

Se realizaron dos ensayos por cada uno de los peldaños estudiados, tomándose la media de ambos como resultado final.

\section{RESULTADOS}

Las microestructuras correspondientes a las muestras obtenidas del nervio central de los peldaños de elevación se muestran en la figura 5. Asimismo, la variación de la fracción en volumen y del tamaño de grano del $\mathrm{Al}-\alpha$, por un lado, y del silicio cuboidal, por otro, están recogidos en la tabla II. Los valores de las fracciones en volumen de los cuboides de silicio resultan similares, observándose un ligero descenso en el tamaño de los mismos en las muestras del peldaño obtenido a partir de la aleación 2 . Por otra parte, los resultados obtenidos muestran diferencias significativas en los tamaños de grano de la fase Al- $\alpha$, siendo el menor el correspondiente al peldaño fabricado a partir de la aleación 2, de tamaño, aproximadamente, mitad del medido en la muestra de la aleación 1.

Las variaciones de las propiedades mecánicas a tracción y los resultados obtenidos tras realizar el ensayo que simula el comportamiento límite en servicio sobre los peldaños se muestran en la tabla III. Los mejores

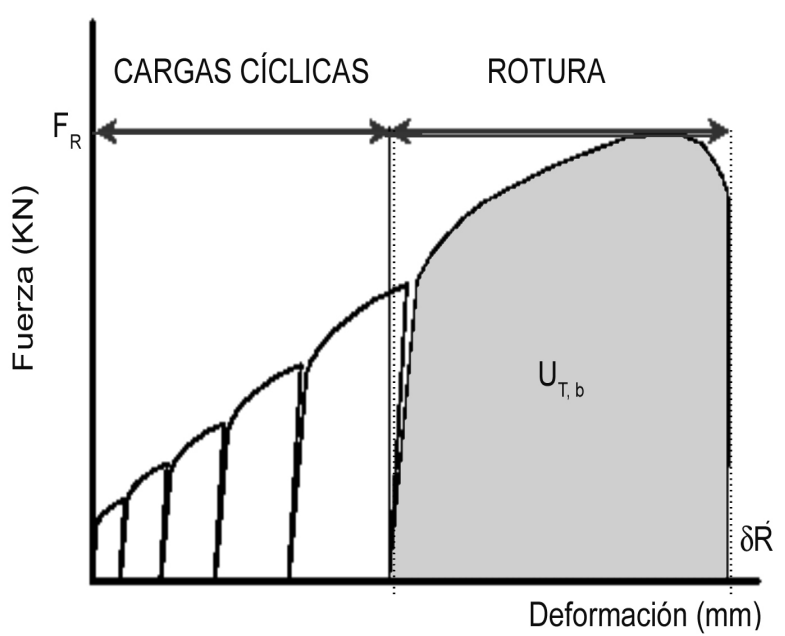

Figura 4. Curva esfuerzos - deformaciones obtenida tras el ensayo de flexión de los peldaños, siendo $U_{\mathrm{T}, \mathrm{b}}$ la energía consumida en la rotura del componente, $\delta_{\mathrm{R}}$ la deformación plástica experimentada por el material tras la rotura y $F_{R}$ la carga de rotura.

Figure 4. Load-displacement curve obtained during the different stages of testing for escalator steps. Keys: $U_{T, b}$ is the total energy to fracture of the component, $\delta_{R}$ the maximum elongation to fracture of the step, and $F_{R}$ the load to rupture.

resultados bajo esfuerzos de tracción se obtuvieron para los ecopeldaños fabricados a partir de la aleación comercial afinada y modificada (aleación 2). El límite elástico y el alargamiento a rotura son 1,8 y 1,4 veces superiores, respectivamente, a los observados en los peldaños convencionales. Los resultados de flexión señalan, al igual que en el ensayo anterior, un mejor comportamiento de los ecopeldaños.

A la hora de valorar cuál de los peldaños presenta un comportamiento mecánico óptimo, se ha optado por un análisis atributivo ${ }^{[15-16]}$ (Tabla IV). Los mejores resultados, bajo todos los supuestos corresponden al ecopeldaño.

\section{DISCUSION}

\subsection{Caracterización microestructural}

Tras someter a las aleaciones objeto de estudio a la técnica de conformado por moldeo a presión, se observa un afino general de las fases presentes (Fig. 5). La microestructura está formada por finos granos de fase Al- $\alpha$ con morfología cuasi-equiáxica, además de cuboides de silicio finos y poco abundantes, sólo 

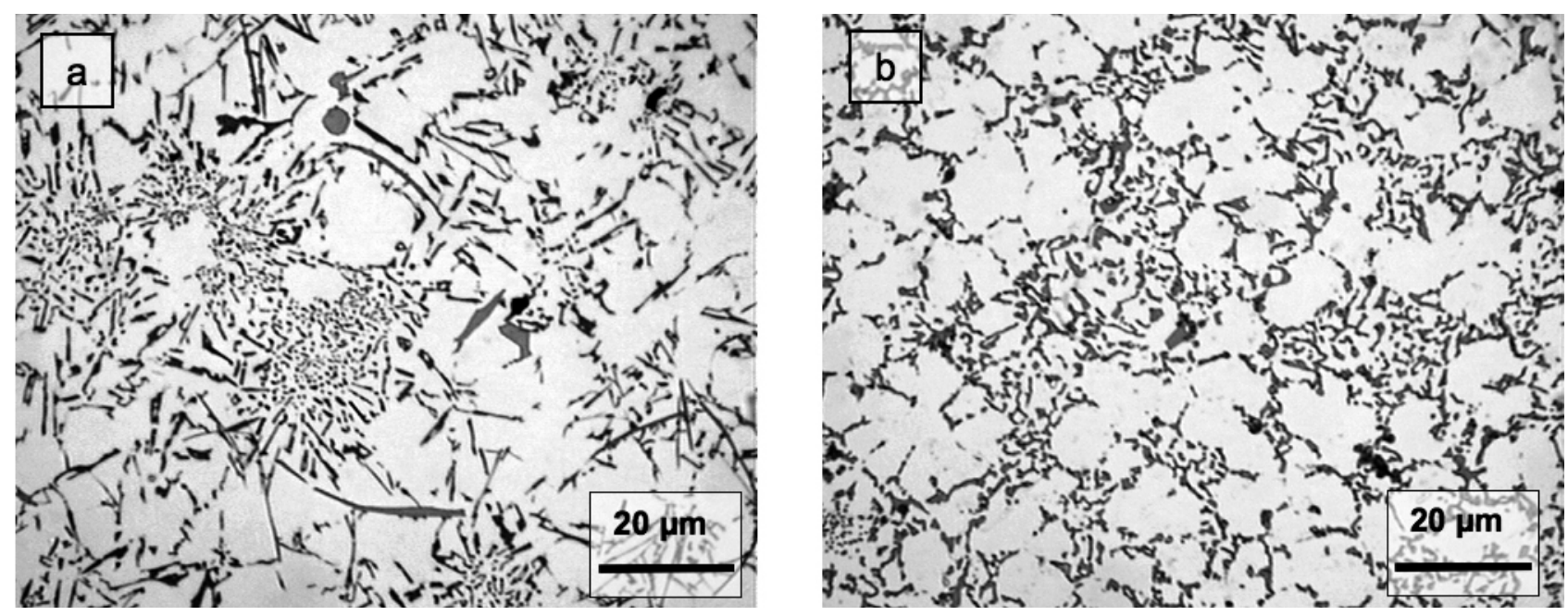

Figura 5. Microestructuras obtenidas utilizando microscopía óptica de reflexión en muestras procedentes de piezas fabricadas mediante fundición a presión: (a) en la aleación 1 se observan granos de fase $\mathrm{Al}-\alpha$ con morfología casi equiáxica, junto con finos cuboides de silicio muy escasos, además de una eutéctica con silicio en forma de finas agujas y (b) las fases presentes son aún más finas en la aleación 2 y la eutéctica presenta una morfología cuasi-globular.

Figure 5. Light optical micrographs of samples cut from pressure die cast components: (a) grained $\alpha-A l$ almost equiaxed in shape, with a fine and scant population of silicon cuboids together with an eutectic with fine Si needles in alloy 1; and (b) considerable refinement of all primary phases and quasiglobular eutectic in alloy 2.

Tabla II. Valores medidos de la fracción en volumen $\left(V_{V}\right)$ y del tamaño para la fase $A l-\alpha\left(\bar{A}_{a}\right)$ y para los cuboides de silicio $\left(\overline{d_{p}}\right)$ en los peldaños de elevación

Table II. Volume fractions $\left(\underline{V}_{v}\right)$ of primary phases, grain size of primary $\alpha-A l\left(\bar{A}_{a}\right)$ and cuboidal silicon particle size $\left(\bar{d}_{p}\right)$ determinations for samples solidified under high pressure conditions in dies

\begin{tabular}{ccccc}
\hline & \multicolumn{2}{c}{$\mathrm{Al}-\alpha$} & \multicolumn{2}{c}{ Cuboides de Si } \\
\cline { 2 - 5 } Aleación & $\begin{array}{c}\mathrm{V}_{\mathrm{v}} \pm \mathrm{CL}_{95 \%} \\
(\%-\mathrm{vol})\end{array}$ & $\begin{array}{c}\overline{\mathrm{A}}_{\mathrm{a}} \pm \mathrm{CL}_{95 \%} \\
\left(\mu \mathrm{m}^{2}\right)\end{array}$ & $\begin{array}{c}\mathrm{V}_{\mathrm{v}} \pm \mathrm{CL}_{95 \%} \\
(\%-\mathrm{vol})\end{array}$ & $\begin{array}{c}\bar{d}_{p} \pm \mathrm{CL}_{95 \%} \\
(\mu \mathrm{m})\end{array}$ \\
\hline 1 & $46,23 \pm 4,14$ & $553 \pm 35$ & $1,23 \pm 0,24$ & $3,7 \pm 0,2$ \\
2 & $63,64 \pm 5,20$ & $212 \pm 19$ & $1,05 \pm 0,16$ & $2,3 \pm 0,1$ \\
\hline
\end{tabular}

visibles a 1.000 aumentos, así como una matriz eutéctica con silicio (Fig. 5).

Atendiendo a las muestras analizadas, el estudio microestructural comparado se estructura como sigue:

\subsubsection{Aluminio a primario}

La morfología de la fase primaria Al- $\alpha$ es similar en los componentes fabricados a partir de ambas aleaciones. Predomina la geometría celular, con muy escasas ramificaciones secundarias (Fig. 5).

En procesos de solidificación a presión, los autores han podido constatar que, en aleaciones eutécticas Al-Si, el estroncio perjudica el poder afinante del titanio sobre el tamaño de la fase Al- $\alpha$ cuando ambos elementos se adicionan simultáneamente ${ }^{[8]}$. En el presente estudio los valores medidos para el tamaño de grano Al- $\alpha$ son 2,6 veces menores. Todo 
Tabla III. Variación de las propiedades mecánicas en los peldaños de elevación

Table III. Mechanical properties variation for the escalator steps

\begin{tabular}{|c|c|c|c|c|c|c|c|c|c|c|c|c|c|c|}
\hline \multirow[b]{3}{*}{ Tipo de peldaño } & \multicolumn{8}{|c|}{ Ensayo de tracción } & \multicolumn{6}{|c|}{ Ensayo de fatiga por flexión } \\
\hline & \multicolumn{2}{|c|}{$R_{p}(M P a)$} & \multicolumn{2}{|c|}{$R_{m}(M P a)$} & \multicolumn{2}{|c|}{$A_{T}(\%)$} & \multicolumn{2}{|c|}{$U_{V, t}\left(M x^{-3}\right)$} & \multicolumn{2}{|c|}{$\mathrm{F}_{\mathrm{R}}(\mathrm{kN})$} & \multicolumn{2}{|c|}{$\delta_{R}(\mathrm{~mm})$} & \multicolumn{2}{|c|}{$U_{T, b}(J)$} \\
\hline & $\overline{R_{p}}$ & $s(x)$ & $\overline{\mathrm{R}_{\mathrm{m}}}$ & $s(x)$ & $\overline{\mathrm{A}_{\mathrm{T}}}$ & $s(x)$ & $\overline{\bar{U}_{v, t}}$ & $s(x)$ & $\overline{\mathrm{F}_{\mathrm{R}}}$ & $s(x)$ & $\overline{\delta_{R}}$ & $s(x)$ & ${\overline{\mathrm{U}_{\mathrm{T}, \mathrm{b}}}}_{\mathrm{s}}$ & $s(x)$ \\
\hline Conv & 65,00 & 5,30 & 141,54 & 2,99 & 0,96 & 0,06 & 135,88 & 3,44 & 23,91 & 1,30 & 13,38 & 0,90 & 186,31 & 0,92 \\
\hline Ecopeld & 113,88 & 3,79 & 167,34 & 4,73 & 1,38 & 0,02 & 254,65 & 3,24 & 29,77 & 1,58 & 29,93 & 2,27 & 581,77 & 3,63 \\
\hline
\end{tabular}

Tabla IV. Propiedades mecánicas en los peldaños de elevación: índices y análisis atributivo

Table IV. Mechanical properties of the escalator steps: indexes and attributive analysis

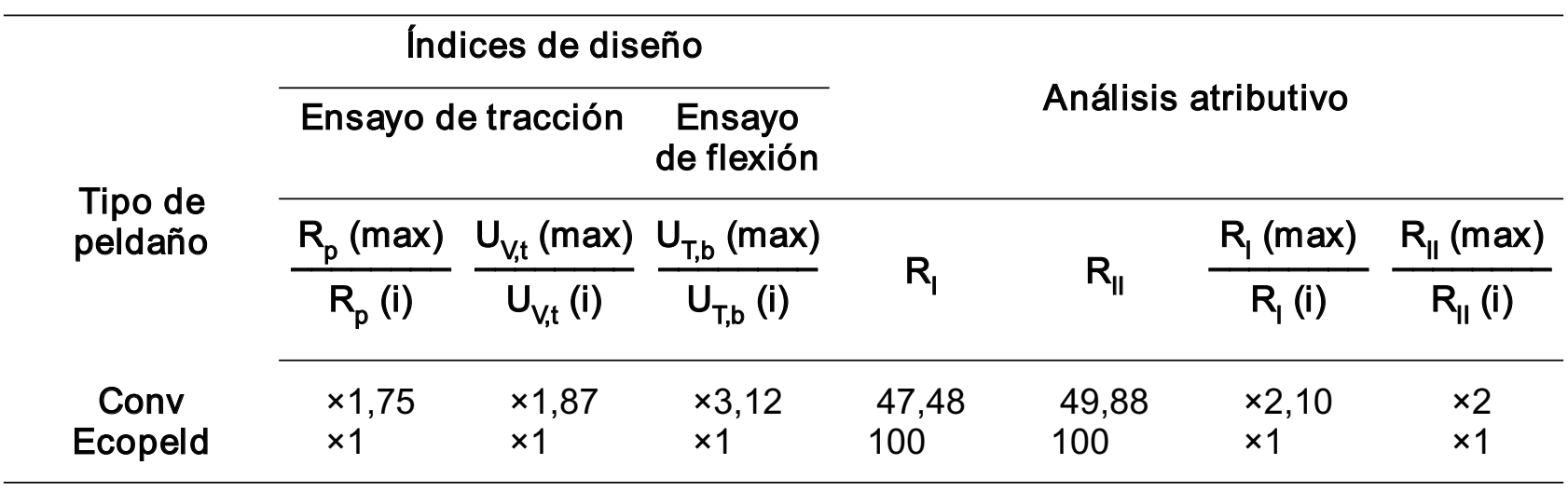

parece indicar que cuando el ratio $\mathrm{Ti} / \mathrm{Sr}=4$ (aleación 2) el fenómeno anteriormente señalado queda obviado frente a aleaciones con ratios, para el cociente de estos elementos, próximas a $1^{[8]}$.

La finura observada en la fase Al- $\alpha$ obedece a las altas presiones que requiere la técnica de colada a presión (del orden de $340 \mathrm{kN} / \mathrm{m}^{2}$ ), que pueden modificar el diagrama de equilibrio. En los metales que se contraen al solidificar, según la ecuación de Claussius-Clapeyron ${ }^{[17]}$, un aumento de presión comporta la elevación de la temperatura de solidificación, afectando a las previsiones sobre las temperaturas de inicio y de final de solidificación, y a las solubilidades de los aleantes en el metal base. El diagrama de solidificación Al-Si cambia sustancialmente ${ }^{[18]}$ : la composición del punto eutéctico, bajo la acción de presiones elevadas, varía su posición desplazándose hacia un mayor contenido en silicio. Dicho aumento de presión ocasiona un aumento de $\approx 5^{\circ} \mathrm{C}$ en la temperatura eutéctica de equilibrio. En definitiva, el efecto de la presión sobre la microestructura se manifiesta mediante un afino y un aumento de la proporción relativa de $\mathrm{Al}-\alpha$.

Además, la elevada presión aplicada durante el proceso de solidificación, tiene un efecto compactador al romper los puentes sólidos entre los dendritos, facilitando la alimentación de líquido residual a los microrrechupes. A la vez, la elevada velocidad de enfriamiento, reduce el espaciado interdendrítico y, por tanto, el nivel de porosidad. Resulta, entonces, que la fracción en volumen de microrrechupes en los peldaños obtenidos mediante moldeo a presión es prácticamente inexistente ${ }^{[19]}$ (Fig. 5).

\subsubsection{Silicio eutéctico y cuboidal}

Lu et al. ${ }^{[20]}$ demostraron, en sus trabajos de investigación, que el estroncio es capaz de producir un elevado número de planos de maclado durante el crecimiento del silicio, modificando su forma y su tamaño. En coincidencia con estos resultados, en el presente trabajo se observa una globulización del silicio eutéctico en 
la muestra fabricada por moldeo a presión a partir de la aleación 2 (Fig. 5 b)). Además, a estas muestras les corresponden los menores tamaños $(2,3 \mu \mathrm{m})$ y fracciones en volumen de cuboides de silicio $(1,05 \%)$ (Tabla II).

\subsubsection{Compuestos intermetálicos}

Se ha observado la presencia de agujas de AlFeSi- $\beta$ en la aleación 1 y no así en la aleación 2, ambas solidificadas bajo presión (Fig. 5). Por ello, todo parece apuntar a que el manganeso pudiese ser el responsable de la no formación de estos compuestos aciculares, incluso, en piezas solidificadas a presión. Lo cual sería posible, a través de la relación Fe/Mn que, en la aleación 2, es muy próxima a la recomendada cuando se pretende estabilizar la variante $\alpha$ del intermetálico ternario $\mathrm{AlFeSi}$, para la que la literatura técnica consultada señala que $\mathrm{Fe} / \mathrm{Mn}=2^{[21]}$.

\subsection{Propiedades mecánicas en tracción}

Las propiedades mecánicas de las aleaciones constituidas por más de una fase vienen determinadas, en primer lugar, por la matriz (eutéctica, en este caso) $\mathrm{y}$, en segundo término, por las fases proeutécticas (Al- $\alpha$ y Si cuboidal).

La deformación plástica en las aleaciones constituidas por fases con distinta dureza $(\mathrm{Al}-\alpha$ y $\mathrm{Si})$ es anisótropa, deformándose más la fase mas blanda (Ala) Esta plasticidad local provoca un rápido endurecimiento de la aleación que conlleva el anclaje de las dislocaciones y la formación de microgrietas en las intercaras $\mathrm{Al}-\alpha / \mathrm{Si}$. El avance de las grietas entre ambas fases se ve favorecido por la baja energía interfacial existente entre ellas. Además, la morfología acicular del silicio en la fase eutéctica induce la concentración de tensiones en los bordes de las agujas, promoviendo la formación de cavidades que, por coalescencia, conducen, finalmente, a la rotura del material. La modificación con estroncio promueve un cambio de morfología en las agujas de silicio eutéctico, que se transforman en glóbulos, mejorando considerablemente las propiedades mecánicas, al desaparecer el efecto concentrador de tensiones ${ }^{[22]}$.

Existe una correlación entre la microestructura de las muestras: el tamaño de la fase Al- $\alpha$ primaria, el tamaño y la forma del silicio cuboidal y eutéctico, etc. (Tabla II) y las propiedades obtenidas a partir de los ensayos mecánicos (Tabla III). Se constata que la modificación de la forma del silicio eutéctico ${ }^{[23]}$, unida a los afinos de tamaño de las fases Al- $\alpha$ y silicio cuboidal (proeutéctico) otorgan, todas ellas, mejoras del comportamiento mecánico de la aleación. En concreto, se consiguen aumentos de la tenacidad estática, resistencia a la tracción y ductilidad. La mejora de las propiedades mecánicas se completa si la distribución de las segundas fases precipitadas resulta uniforme en todo su volumen ${ }^{[24]}$.

Analizando los resultados obtenidos tras ensayar probetas de tracción planas extraídas del nervio central de los ecopeldaños y comparándolos con los obtenidos en los peldaños convencionales se observa (Tabla III) que:

- El límite elástico es 1,8 veces mejor. Este resultado puede ser atribuido, por un lado, al efecto afinador del titanio y a una mayor fracción en volumen de Al- $\alpha$ y, por otro lado, a la modificación de la forma del silicio eutéctico, debido a la acción del estroncio y a la elevada presión aplicada.

- La carga de rotura se ve mejorada con morfologías eutécticas finas y globulares que evitan la concentración de tensiones y provocan un descenso del área superficial por unidad de volumen, favoreciendo la distribución isotrópica de las tensiones ${ }^{[2]}$. La carga de rotura también aumenta cuanto mayor es la fracción en volumen y menor el tamaño de grano de la fase $\mathrm{Al}$ $\alpha$. Todo ello justifica que la carga de rotura de los ecopeldaños supere en 25,8 MPa a la obtenida en los peldaños convencionales.

- La ductilidad alcanza niveles óptimos minimizando aquellos factores que propicien la aparición de poros y microrrechupes, y maximizando los que ayuden a frenar el avance de las grietas ${ }^{[25]}$. Será necesario desarrollar microestructuras libres de elementos concentradores de tensiones, como el silicio eutéctico laminar y el silicio cuboidal, y retardar la propagación de las grietas mediante el aumento de la fracción en volumen y la disminución del tamaño de la fase plástica Al- $\alpha$. Esta fase, más blanda y con un límite elástico inferior que el silicio, proporciona un sistema de confinamiento plástico al avance de las grietas mediante el clásico mecanismo de enromamiento ${ }^{[26]}$. En línea con estos razonamientos se encuentra el hecho de que los mejores resultados en cuanto a ductilidad se encontraron en los ecopeldaños.

\subsection{Ensayo de flexión en tres puntos}

El ecopeldaño resulta el componente más tenaz en el ensayo estático de flexión a rotura, obteniendo un 
valor de la energía de rotura de $582 \mathrm{~J}$ en el ecopeldaño frente a $186 \mathrm{~J}$ en el peldaño convencional (Tabla III). En este sentido, es conveniente resaltar el rol del manganeso, que inhibe la formación de agujas de la fase $\beta^{[27-29]}$, aminorando la microfisuración entre la matriz y las agujas de la fase $\beta$. Todo parece indicar que la ausencia de agujas de este compuesto intermetálico, su menor fracción en volumen y el menor tamaño de los cuboides de silicio, unidos a la mayor fracción en volumen de Al- $\alpha$, junto con la finura de ésta (Tabla II) pudieran ser los responsables de que la flecha a rotura sea 2,2 veces mayor, que la $F_{R}$ a rotura sea 1,25 veces mayor $y$, finalmente, que la energía absorbida antes de la rotura resulte 3,12 veces superior en el ecopeldaño.

\subsection{Criterios de diseño de componentes}

Los resultados obtenidos en estos ensayos posibilitan el planteamiento de un proceso de selección que permita determinar el material óptimo, teniendo en cuenta la función que desempeñarán los peldaños de elevación a lo largo de su vida útil.

\subsubsection{Diseño de componentes sin deformación plástica}

Un primer criterio de selección consiste en diseñar un peldaño con el valor de resistencia a la deformación plástica a tracción lo más elevado posible. Se intenta evitar la deformación plástica del componente, quedando el proceso de selección reducido a la búsqueda de aquel material con más alto límite elástico[30]. Los resultados de la tabla III y el índice $R_{p}(\max ) / R_{p}(i)$ de la tabla IV, muestran que el ecopeldaño de elevación es el que optimiza este criterio de selección, al ser 1,75 veces mejor el ecopeldaño (aleación 2) que el peldaño convencional (aleación 1).

\subsubsection{Diseño de componentes con deformación plástica}

Si el criterio de selección admite la deformación plástica del componente, éste debe ser capaz de absorber gran cantidad de energía y deformarse plásticamente antes de romper. Se trata de evitar provocar daños a personas y/ó equipos (criterio "avisa" antes de romper). A la hora de valorar este criterio de selección, deben considerarse los principales esfuerzos a los que estará sometido el peldaño a lo largo de su vida útil:

- Las fibras del peldaño situadas por debajo del plano neutro (Fig. 1) estarán sometidas a esfuerzos de tracción. Su comportamiento puede ser ponderado mediante la medida del área bajo la curva ingenieril de tracción, que proporciona la energía absorbida por el material antes de la rotura, $\mathrm{U}_{\mathrm{V}, \mathrm{t}}$, (Tabla III). El índice $\mathrm{U}_{\mathrm{V}, \mathrm{t}}(\max ) / \mathrm{U}_{\mathrm{V}, \mathrm{t}}(\mathrm{i})$ cuantifica los valores más elevados. Estos corresponden a los ecopeldaños (Tabla IV), que mejoran en un $87 \%$ el valor obtenido para el peldaño convencional.

- El área encerrada bajo la curva F- $\delta$ en el ensayo de flexión a rotura (Fig.4) señala la energía absorbida por el peldaño antes de la rotura, $\mathrm{U}_{\mathrm{T}, \mathrm{b}}$. Ésta energía corresponde a los peldaños de elevación rotos en condiciones de flexión, después de provocarles un daño mediante fatiga de bajo número de ciclos. La tabla III proporciona los valores de energía calculados a partir de las curvas F-d para cada peldaño ensayado. Los resultados de la tabla III y del índice $\mathrm{U}_{\mathrm{T}, \mathrm{b}}(\max ) /$ $\mathrm{U}_{\mathrm{T}, \mathrm{b}}(\mathrm{i})$ de la tabla IV, ponen nuevamente de manifiesto que el ecopeldaño de elevación es el que optimiza este criterio de selección.

\subsection{Análisis atributivo para seleccionar el peldaño con las mejores propiedades mecánicas}

A la hora de valorar cuál de los peldaños presenta un comportamiento mecánico óptimo, se ha optado por un análisis atributivo ${ }^{[31-34]}$ en el que se maximizan las propiedades mecánicas que aparecen en la tabla III: la resistencia a la deformación plástica bajo esfuerzos de tracción, $R_{p}$, la energía total absorbida en tracción, $U_{V, t}$ y la energía absorbida bajo esfuerzos de flexión, $\mathrm{U}_{\mathrm{T}, \mathrm{b}}$, . Se proponen a continuación las siguientes hipótesis:

- Un primer análisis atributivo que concediese igual importancia a estas propiedades mecánicas nos llevaría a la ecuación 1 :

$$
R_{1}(i)=100 \times\left[\frac{1}{3}\left(\frac{R_{p}(i)}{R_{p}(\max )}\right)+\frac{1}{3}\left(\frac{U_{V, t}(i)}{U_{V, t}(\max )}\right)+\frac{1}{3}\left(\frac{U_{T, b}(i)}{U_{T, b}(\max )}\right)\right]
$$

- En la realidad los peldaños se remplazan antes de romper, por lo que un análisis atributivo más realista sería el que concediese mayor importancia al comportamiento elástico frente al elasto-plástico. Los coeficientes de peso se- 
UNA ESTRATEGIA DE ECODISEÑO DE PIEZAS OBTENIDAS MEDIANTE MOLDEO A PRESIÓN: ANÁLISIS MICROESTRUCTURAL APLICADO A LA DESMATERIALIZACIÓN AN ECODESIGN STRATEGY FOR HIGH PRESSURE DIE CASTING COMPONENTS: MICROSTRUCTURAL ANALYSIS APPLIED TO MASS REDUCING PROCESSES

rían: un $50 \%$ al comportamiento elástico en tracción, un $25 \%$ a la energía elástico - plástica absorbida en tracción hasta la rotura y un $25 \%$ al comportamiento elasto-plástico a rotura en flexión. En resumen, este nuevo análisis proporciona un $50 \%$ de peso al comportamiento elástico y el $50 \%$ restante al comportamiento elasto-plástico hasta la rotura, resultando finalmente la ecuación 2:

$$
R_{\| l}(i)=100 \times\left[\frac{1}{2}\left(\frac{R_{p}(i)}{R_{p}(\max )}\right)+\frac{1}{4}\left(\frac{U_{V, t}(i)}{U_{V, t}(\max )}\right)+\frac{1}{4}\left(\frac{U_{T, b}(i)}{U_{T, b}(\max )}\right)\right](2)
$$

A la vista de los resultados relativos a las propiedades mecánicas de los peldaños de elevación (Tabla IV), queda patente que los mejores resultados en ambos supuestos se obtienen en los ecopeldaños. Y, con independencia de las hipótesis señaladas, el ecopeldaño resulta dos veces mejor que el peldaño convencional.

\section{CONCLUSIONES}

Se ha probado la posibilidad de fabricar ecopeldaños de elevación con destino a escaleras mecánicas, menos reforzados estructuralmente y, por tanto, más ligeros que los peldaños convencionales que hasta la fecha se venían fabricando mediante idéntico proceso productivo.

Se constata que la solidificación a presión efectúa modificaciones del diagrama de fases $\mathrm{Al}-\mathrm{Si}$, que acentúan el carácter hipoeutéctico de las aleaciones eutécticas y ligeramente hipoeutécticas.

La relación $\mathrm{Fe} / \mathrm{Mn}=2$ es relevante a fin de inhibir la formación de la fase acicular del intermetálico AlFeSi a presión elevada.

Los resultados experimentales muestran que los ecopeldaños fabricados con una aleación comercial afinada y modificada mejoran los obtenidos en un peldaño convencional tanto en tracción como en flexión. Además, un análisis atributivo permitió constatar que bajo la acción combinada de esfuerzos y ponderando tanto el comportamiento elástico como el elasto-plástico, los resultados en el ecopeldaño son dos veces mejores que en el peldaño convencional.

A la vista de los resultados se puede concluir señalando que es posible reducir el uso de materiales durante la fabricación mediante colada a presión, de los peldaños de elevación y disminuir el impacto a lo largo de su vida útil, todo ello mejorando las prestaciones en servicio.

\section{REFERENCIAS}

[1] A. Aranda, I. Zabalza y A. Martinez, El Análisis del Ciclo de Vida como Herramienta de Gestión Empresarial, Fundación Confemetal, 2006. pp. 27-37.

[2] J. R. Brown, Foseco Non-ferrous Foundryman's Handbook, Butterworth- Heinemann, Oxford, 1999.

[3] S. K. Kim, H. Cho, H. Jo, M. Han, S. Lim y T. Hur, Mater. Sci. Forum 426-432, 3.353-3.358.

[4] B. Neto, C. Kroeze, L. Hordijk, C. Costa y T. Pulles, J. Environ. Manage. (2008) 1-16.

[5] B. Neto, C. Kroeze, L. Hordijk, C. Costa y T. Pulles, Environ. Modell. Softw. 23 (2008) 147-168.

[6] A. C. K. Choi, H. Kaebernick y W. H. Lai, J. Mater. Process. Technol. 70 (1997) 231-238.

[7] Aluminium and Aluminium Alloys, A.S.M. Internacional Materials Park, OH, 1994, pp. 26.

[8] J. Asensio-Lozano y B. Suárez-Peña, Mater. Charact. 56 (2006) 169-177.

[9] B. Suárez-Peña, J. Asensio-Lozano, J. I. VerdejaGonzalez y J. A. Pero-Sanz Elorz, Rev. Met. Madrid 43 (2007) 352-358.

[10] J. Muirhead, J. Cawley, A. Strang, C. A. English y J. Titmarsh, Mater. Sci. Technol. 16 (2000) 1.160-1.166.

[11] G. F. Vander Voort, Precision and Reproductivity of Quantitative Measurements, ASM International Materials Park, OH, 1994, pp. 31-34.

[12] R. L. Higginson y C. M. Sellars, Worked examples in quantitative metallography, Institute of Metals, Maney Publishing, London, Inglaterra, 2003.

[13] J. Asensio, Curso de Metalografía Cuantitativa, E. T. S. I. M. O., Oviedo, España, 1992.

[14] ASTM E8, Standards Test Methods of Tension Testing of Metallic Materials. Annual Book of ASTM Standards. 1990. p. 130.

[15] M. F. Asbhy, Materials Selection in Mechanical Design, Pergamon Press, Oxford, 1993, pp. 66-69.

[16] J. A. Pero-Sanz, Ciencia e Ingeniería de Materiales, C. I. E. Inversiones Editoriales - Dossat 2000, Madrid, 2000, pp. 606-608.

[17] Dehoff R. T. Thermodynamics in Materials Science, International Editions McGraw-Hill, Singapore, 1993, p. 142.

[18] B. Suarez-Peña, J. Asensio-Lozano y G. F. Vander Voort Microsc. Microanal. 13 (2007) 1.030 1.031 .

[19] J.E. Gruzsleski y B. M. CLOSSET, The Treatment of Liquid Al-Si alloys. AFS, Des Plaines, (IL) 1990, p. 110.

[20] S. Lu y A. Hellawell, Metall. Trans. A., 18 (1987) 1.721-1.733. 
[21] F. Mondolfo, Aluminum alloys: Structure and Properties, London, Butterworth, 1976, pp. 534776.

[22] G.E. Dieter, Strengthening mechanisms, McGraw Hill, Singapore, 1988.pp. 208-12.

[23] J.A. Garcóa-Hinojosa, C.R. González, G.M. González e Y. Houbaert, J. Mater. Process. Technol 143 (2003) 306-310.

[24] H. Liao, Y Sun y G. Sun, J. Mater. Sci. 37 (2002) 3489-3495.

[25] M. F. Ashby y R. H. Jones, Engineering Materials 1 (second edition), Pergamon Press, Exeter, UK, 1996 p. 77.

[26] M. F. Ashby y R. H. Jones, Engineering Materials 1 (second edition), Pergamon Press, Exeter, UK, 1996 p. 131.

[27] K. V. Kral, H. R. Mcintyre y M. J. Smillie, Scr. Mater. 51 (2004) 215-219.
[28] C. M. Dinnis, J. A. Taylor y A. K. Dahle Scr. Mater. 53 (2005) 955-958.

[29] B. Suarez-Peña y J. Asensio-Lozano Scr. Mater. 54 (2006) 1543-1548.

[30] M. F. Ashby y R. H. Jones, Engineering Materials 1 (second edition), Pergamon Press, Exeter, UK, 1996 p. 261.

[31] M. F. Asbhy, Materials Selection in Mechanical Desing, Pergamon Press, Oxford, 1993, pp. 6669.

[32] J. A. Pero-Sanz, Ciencia e Ingeniería de Materiales, C. I. E. Inversiones Editoriales- Dossat 2000, Madrid, 2000, pp. 606-608.

[33] B. Suárez-Peña y J. Asensio-Lozano. Mater. Charact. 57 (2006) 218-226.

[34] B. Suarez-Peña, J. Asensio-Lozano, J.I. VerdejaGonzalez y J.A. Pero-Sanz. Rev Metal. Madrid 43 (2007) 310-317. 